\title{
Modal analysis and nonlinear characterization of an airborne power ultrasonic transducer with rectangular plate radiator
}

\author{
R.R. Andrés ${ }^{a}$, V.M. Acosta ${ }^{a}$, M. Lucas ${ }^{b}$, E. Riera ${ }^{a}$. \\ a Departamento de Sensores y Sistemas Ultrasónicos (DSSU), ITEFI, CSIC, Serrano 144 \\ 28006, Madrid, Spain \\ b School of Engineering, University of Glasgow, Glasgow G12 8QQ, UK
}

\begin{abstract}
.-
Some industrial processes like particle agglomeration or food dehydration among others can be enhanced by the use of power ultrasonic technologies. These technologies are based on an airborne power ultrasonic transducer (APUT) constituted by a pre-stressed Langevin-type transducer, a mechanical amplifier and an extensive plate radiator. In order to produce the desired effects in industrial processing, the transducer has to vibrate in an extensional mode driving an extensive radiator in the desired flexural mode with high amplitude displacements. Due to the generation of these high amplitude displacements in the radiator surfaces, non-linear effects like frequency shifts, hysteresis or modal interactions, among others, may be produced in the transducer behavior. When any nonlinear effect appears, when applying power, the stability and efficiency of this ultrasonic technology decreases, and the transducer may be damaged depending on the excitation power level and the nature of the nonlinearity. In this paper, an APUT with flat rectangular radiator is presented, as the active part of an innovative system with stepped reflectors. The nonlinear behavior of the APUT has been characterized numerically and experimentally in case of the modal analysis and experimentally in the case of dynamic analysis. According to the results obtained after the experiments, no modal interactions are expected, nor do other nonlinear effects.
\end{abstract}

Keywords: Airborne power ultrasound, ultrasonic transducers, modal analysis, non-linear effects, finite element analysis 


\section{1. - Introduction}

The application of airborne ultrasonic technologies has demonstrated improvements in a number of industrial processes. Among them, processes such as defoaming, particle agglomeration and food dehydration are enhanced if they are assisted by airborne power ultrasound (APU) [1-3], by an increase in efficiency, lower energy consumption and better quality of the final product as in the case of food dehydration $[4,5]$.

In order to produce the desired effects, an airborne power ultrasonic transducer (APUT) has to generate a high intensity ultrasonic field in a gas medium. It implies to cover specific requirements such as good impedance matching between the ultrasonic source and the low-density medium for efficient energy transmission; high directional radiation for energy concentration; high-Q transducers with mechanical amplification for large displacements; and a well-balanced distribution of mechanical stresses in the radiator for operation at maximum power capacity. The impedance matching can be achieved by increasing the radiating surface of the transducer because the radiation impedance is proportional to the surface area of the radiator. In addition, it is important to take into account that, if the dimensions of the radiator are much larger than the wavelength, the radiator will not act as a piston but will vibrate in one of its natural flexural modes and, consequently, the generated acoustic field will not be coherent. The addition of halfwavelength steps in the profile of the plate radiator enables the coherent ultrasonic radiation from an extensive radiator vibrating in a flexural mode [6].

The generation of a high intensity ultrasonic field requires the transducer to vibrate in a tuned operational mode with large displacement, needing a high excitation level. Previous research has shown that this can result in the transducer vibrating in a nonlinear regime, where undesired nonlinear responses can be excited [7,8]. Some of these responses are due to the piezoelectric ceramics experiencing high levels of strain and temperature increases [9], causing frequency shifts [10] or hysteretic responses [11]. Nonlinear responses in the form of resonance frequency drift related to temperature increases can be controlled to some extent by modifying the excitation signal, for example by applying burst instead of continuous excitation [12], but this has only been achieved successfully in lab based measurements to characterize the nonlinear responses and not for controlling the high-power operating excitation conditions. Other nonlinear behaviors, such as multi-modal responses, modal interactions and autoparametric vibrations may also be exhibited in ultrasonic devices at high excitation levels $[13,14]$. Modal interactions may appear as one or more modal responses at frequencies other than the operating one and tend to result in reduced ultrasonic displacement amplitude at the desired operating resonance frequency, even when the ultrasonic generator is driving only at that.

When designing an APU transducer the shape of the stepped plate radiator affects in the behavior of the airborne ultrasonic transducer. Compared to the stepped circular plate, the rectangular plate shows a more uniform distribution of vibration displacements; nevertheless, rectangular plates can also be prone to modal interactions. The non-axisymmetrical shape of the plate results in a complicated vibrational behavior, with high modal density, which is known to facilitate both modal coupling and nonlinear modal interactions [14].

Previous research has investigated these difficulties associated with the use of stepped rectangular plates. Fig 1.a displays a titanium rectangular plate with steps in both faces and with a 14 nodal lines operational mode, designed for an excitation level of $350 \mathrm{~W}$. Energy leakage to harmonics and subharmonics appeared when applying $200 \mathrm{~W}$ [14]. Fig 1.b shows the vibration spectrum of the plate driven at its operating frequency $(20.9 \mathrm{kHz})$ and at a high power regime (200W), where this energy leakage to undesired frequencies can be observed. 


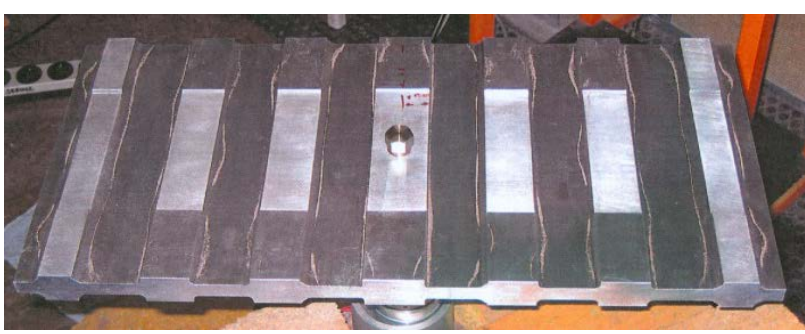

a)

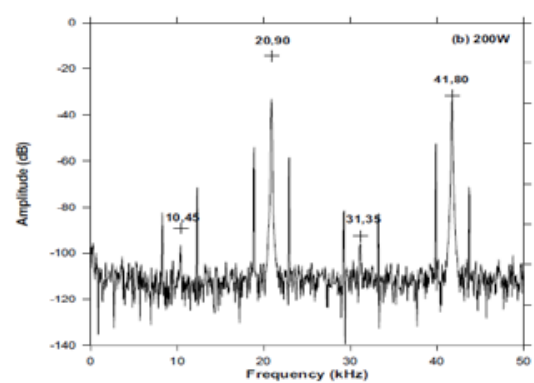

b)

Fig.1. a) Stepped rectangular plate with operational failures. b) Vibration spectrum at $200 \mathrm{~W}$ of the stepped rectangular plate transducer [14].

In order to avoid these effects, rectangular plate transducers should have a pure resonant-mode behavior with a very high quality factor and narrow bandwidth that minimizes modal coupling and energy leakages to undesired modes. The APU transducer with flat rectangular plate introduced in this paper has therefore been designed and built with the main objective of fulfilling these previous requirements.

A new APU transducer has been developed in order to assist mass transfer processes intensification for the food industry, based on previous experimental studies that have demonstrated the efficiency of APU in the enhancement of low temperature dehydration processes $[15,16]$. The development of this technology can be divided into three stages: (1) a theoretical design applying the constitutive equations [17-19], (2) a numerical analysis using a finite element model [20-22], and (3) an experimental characterization at low and high power operation including modal analysis, stability tests and nonlinear behavior [23-25].

We have improved the design of the whole radiating system. The innovations use the total energy emitted by both sides of the plate and places the stepped profiles in the structure of a reflector system instead of on both sides of the plate radiator, as it was firstly presented in [26] for a rectangular plate transducer with an operational frequency of $9.7 \mathrm{kHz}$.

This paper focuses on the active part of this innovative system, the ultrasonic plate transducer and its specific characteristics for the generation of directive or coherent high intensity ultrasonic waves in a gas media.

\section{2. - Description of the APU transducer with flat rectangular plate radiator for mass transfer process intensification}

The APUT basically consists of a piezoelectric Langevin-type transducer with a stack of four piezoceramic rings sandwiched with two metallic rods under an applied prestress of $25 \mathrm{MPa}$, a half-wavelength mechanical amplifier (or horn) to amplify the displacement amplitude. The flat rectangular extensive radiator enables a good impedance matching with the gas medium and high amplitude displacements on the surface of the plate when applying power. To obtain high intensity ultrasonic fields, the radiation should be conveyed to put it all in phase. To that purpose a system of reflectors will be attached to the flat plate radiator as will be described later. The desired operational mode of the transducer corresponds with an extensional mode of the ultrasonic vibrator (composed by the Langevin transducer and the mechanical amplifier) which drives the radiator into resonance in one of its flexural modes. In the present study, the plate radiator vibrates in a flexural mode with twelve nodal lines $(12 \mid 0)$. This resonant mode is excited at a frequency close to $21 \mathrm{kHz}$. The plate radiator transducer is shown in Fig.2, where it can be observed that the piezoelectrically activated ultrasonic vibrator is attached at the plate center. 


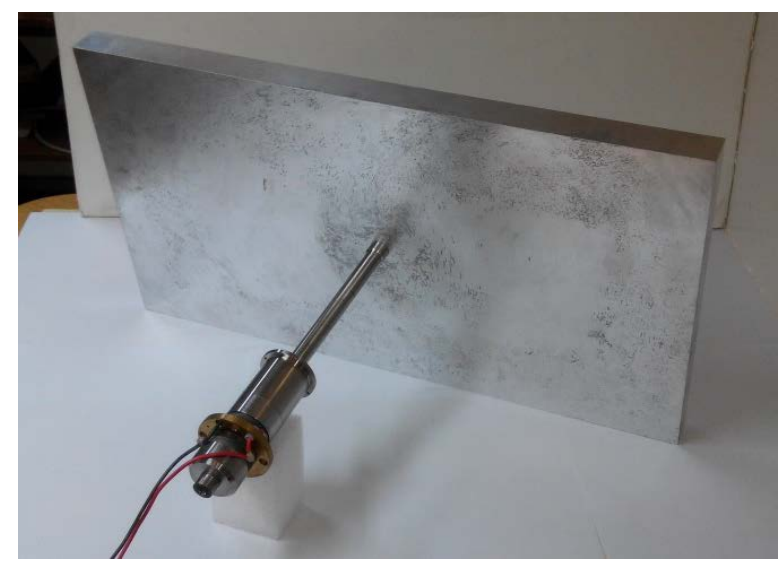

Fig.2. Airborne power ultrasonic transducer with a rectangular plate radiator

The Langevin-type sandwich transducer is composed of two steel cylinders to form the front mass and the back mass. Steel is used because it can withstand the stresses without damage to the internal threads. Four PZT-802 (MTC Electroceramics) ceramic rings are held between the steel masses by a pre-stress bolt. The PZT-802 is chosen due to its high $\mathrm{Qm}_{m}(\approx 2000)$ with moderate generation of heat in order to minimize nonlinear effects and losses.

The electrodes for the electric insulation and connection of the piezoelectric ceramics of the stack are made of copper-beryllium while the central flange is made of brass as it is a good heat conductor and mechanically resistant to support the case covering the sandwich and mechanical amplifier.

The mechanical amplifier provides sufficient displacement amplitude at the tip, where the plate radiator is attached. It consists of two sections, each of quarter-wavelength, where the amplification depends on the ratio of the cross-sectional areas of the bars. For this amplifier, in order to improve the performance of the whole system of plate radiator transducer and reflectors, the mechanical amplifier has been lengthened a half-wavelength in the narrow section. The amplifier is made of a titanium alloy. There are two critical points where failure can occur, one is the union between the amplifier and the plate, consisting of a bolt made of titanium that passes through the plate with a washer between these two elements and is threaded into the horn, and the other is at the cross-section where the amplifier's diameter changes. Titanium allows high mechanical stresses [27] and has moderate internal losses, high $\mathrm{Q}_{m}$ and high efficiency.

The plate radiator is made of an aluminum alloy, in order to minimize mechanical losses, providing good acoustic coupling with the mechanical amplifier. The choice of an aluminum alloy for the plate instead of a titanium alloy is determined by the higher displacement amplitudes and the better mechanical coupling with the mechanical amplifier obtained with the aluminum alloy.

In order to design an efficient system, the size of the rectangular plate $(570 \times 308 \times 34 \mathrm{~mm})$ has to fulfill some requirements. First of all, the power capacity has to be high to generate a high intensity ultrasonic field. Also, the covered area of this ultrasonic field has to be large enough to place sufficient samples to dehydrate. The length and width of the plate fulfill the requirements, and build a plate with practical size, easy to work with.

Regarding the thickness of the plate, a theoretical method is commonly used for thin plates [2830]. Nevertheless, the designed plate is considered as a thick plate, so its final geometry has been established numerically, by FEM, considering the mode $12 \mid 0$ at $21 \mathrm{kHz}$ and the length $(570 \mathrm{~mm})$ and width $(308 \mathrm{~mm})$ as initial parameters. The numerical model, that has been validated experimentally, determines the thickness to obtain the desired vibration mode and gives information about other near modes that may affect the final performance of the system. 
The electrical properties of the APU transducer, measured using an impedance analyzer (HP 4194A), are shown in Table 1, where the values of the bandwidth and the quality factor indicate a highly resonant system. The Bode plot with the admittance $(Y)$ of the transducer, including the real part (Conductance $(G)$ ) and the imaginary part (Susceptance (B)) is shown in Fig. 3, where the bandwidth can be observed

Table 1. Electrical properties of the transducer.

\begin{tabular}{|c|c|c|c|c|c|}
\hline Frequency (Hz) & $\begin{array}{c}\text { Conductance G } \\
(\mathbf{m S})\end{array}$ & $\begin{array}{c}\text { Impedance IZI } \\
(\mathbf{\Omega})\end{array}$ & $\begin{array}{c}\text { Bandwidth } \\
(\mathbf{H z})\end{array}$ & $\begin{array}{c}\text { Efficiency } \boldsymbol{\eta} \\
(\%)\end{array}$ & $\begin{array}{c}\text { Quality } \\
\text { Factor } \mathbf{Q}\end{array}$ \\
\hline 21097 & 1.60 & 625 & 1.59 & 80 & 13268 \\
\hline
\end{tabular}

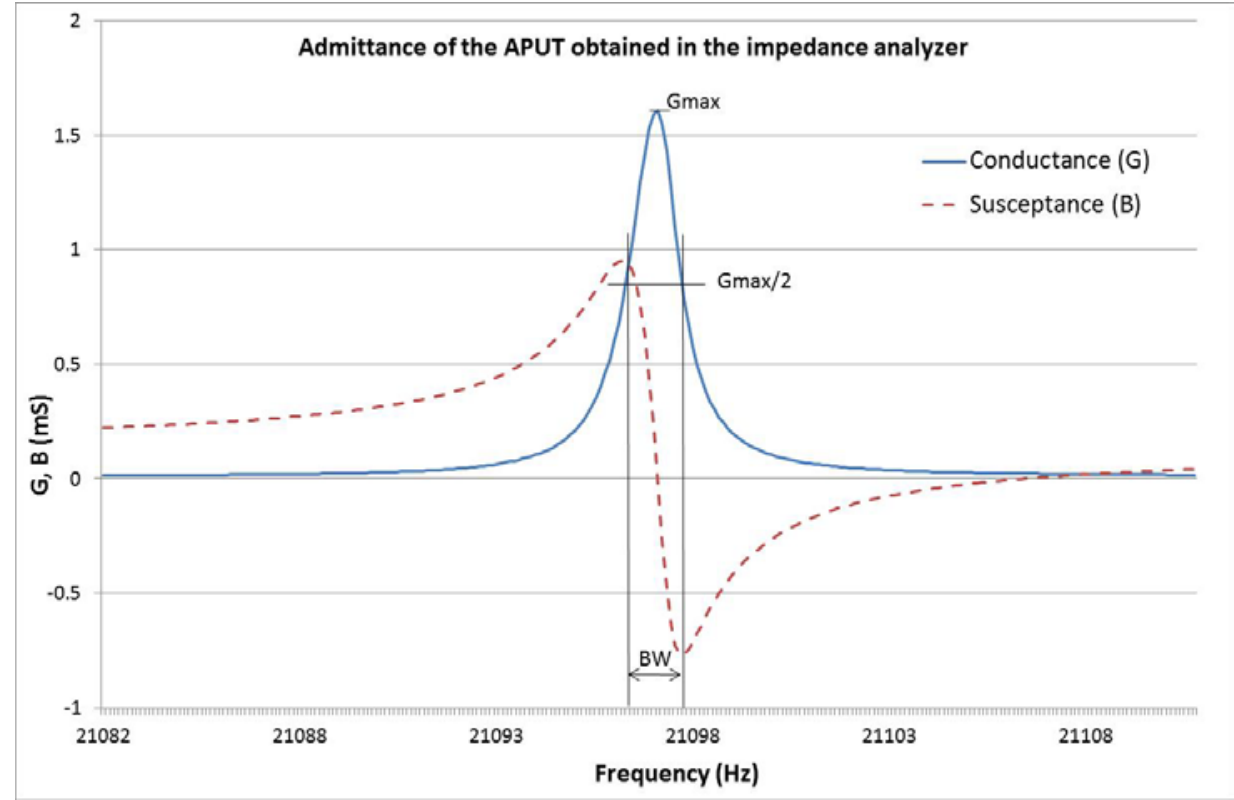

Fig.3. Electrical response of the airborne power ultrasonic transducer,

\section{3. - Modal analysis of the airborne power ultrasonic transducer}

\section{1. - Numerical analysis}

The numerical analysis was carried out with the FEM software COMSOL Multiphysics ${ }^{\circledR}$. The objective was to design a transducer with a rectangular plate radiator vibrating in an operational mode of twelve nodal lines (12NL) at a frequency around $21 \mathrm{kHz}$.

As aforementioned, the piezoelectric properties of the PZT-802 ceramics are contained in Equations 1-3, and the mechanical properties of the flange, masses, titanium alloy amplifier and aluminum alloy flat rectangular plate radiator are provided in Table 2.

The elasticity matrix $\left[c^{E}\right]$ of the PZT-802 piezoelectric ceramic rings is given by:

$$
\left[c^{E}\right]=\left[\begin{array}{cccccc}
1.47 \mathrm{e} 11 & 8.11 \mathrm{e} 10 & 8.11 \mathrm{e} 10 & 0 & 0 & 0 \\
8.11 \mathrm{e} 10 & 1.47 \mathrm{e} 11 & 8.11 \mathrm{e} 10 & 0 & 0 & 0 \\
8.11 \mathrm{e} 10 & 8.11 \mathrm{e} 10 & 1.32 \mathrm{e} 11 & 0 & 0 & 0 \\
0 & 0 & 0 & 3.13 \mathrm{e} 10 & 0 & 0 \\
0 & 0 & 0 & 0 & 3.13 \mathrm{e} 10 & 0 \\
0 & 0 & 0 & 0 & 0 & 3.29 \mathrm{e} 10
\end{array}\right] \mathrm{Pa}
$$

The coupling matrix [e] of the PZT-802 piezoelectric ceramic rings is given by: 


$$
[e]=\left[\begin{array}{ccc}
0 & 0 & -4.1 \\
0 & 0 & -4.1 \\
0 & 0 & 14 \\
0 & 10.3 & 0 \\
10.3 & 0 & 0 \\
0 & 0 & 0
\end{array}\right] \mathrm{C} / \mathrm{m}^{2}
$$

The dielectric permittivity matrix at constant strain $\left[\epsilon_{\mathrm{T}}^{\mathrm{S}}\right]$ of the PZT-802 piezoelectric ceramic rings is given by:

$$
\left[\epsilon_{T}^{S}\right]=\left[\begin{array}{ccc}
900 & 0 & 0 \\
0 & 900 & 0 \\
0 & 0 & 600
\end{array}\right]
$$

Table 2. Mechanical properties of the constitutive parts of the transducer.

\begin{tabular}{|c|c|c|c|c|}
\hline & Brass flange & $\begin{array}{c}\text { Steel back and } \\
\text { front masses }\end{array}$ & $\begin{array}{c}\text { Titanium } \\
\text { mechanical } \\
\text { amplifier }\end{array}$ & $\begin{array}{c}\text { Aluminum } \\
\text { rectangular plate }\end{array}$ \\
\hline Density [kg/m $\left.{ }^{3}\right]$ & 8350 & 7715 & 4423 & 2760 \\
\hline $\begin{array}{c}\text { Young's modulus } \\
{[\mathrm{GPa}]}\end{array}$ & 92.7 & 218.7 & 117 & 70.7 \\
\hline Poisson's ratio & 0.365 & 0.28 & 0.32 & 0.341 \\
\hline
\end{tabular}

The APUT has to operate in a specific mode with high amplitude vibration displacements. As mentioned in the introduction, transducers with rectangular shaped radiators can exhibit many eigenmodes at frequencies close to the tuned modal frequency, which could be giving rise to modal coupling and loss of stability at high power

A numerical modal analysis is therefore carried out to identify the modal frequencies and the mode shapes. The modal analysis is performed as a 3D FEM and under resonance (or "short circuit") conditions [21].The desired resonance mode consists on an extensional mode of the vibrator and a $12 \mathrm{NL}$ flexural mode of the rectangular radiator.

The results of the numerical modal analysis of the plate radiator transducer are shown in Figs. 45. Fig 4.a shows the operational mode, with an extensional mode in the vibrator and the twelve nodal lines mode in the plate. The modal frequency of the full transducer in this case is $21049 \mathrm{~Hz}$.

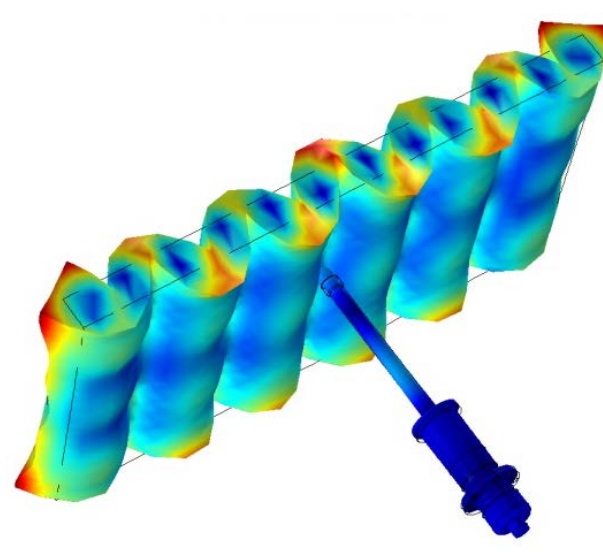

a)

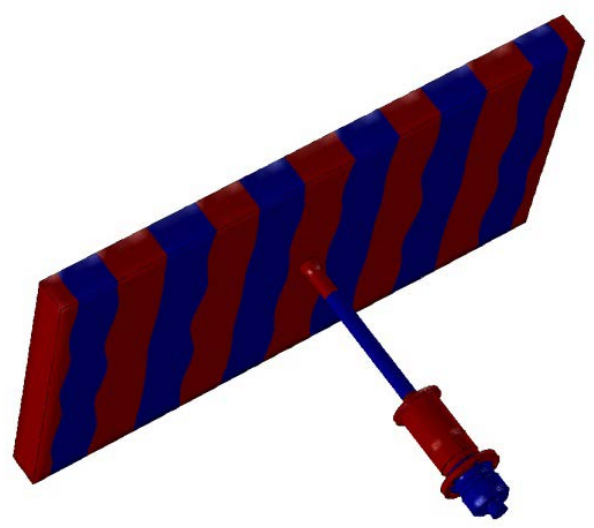

b)

Fig.4. Operational mode at $21049 \mathrm{~Hz}$. a) Mode shape with 12NL. b) Node distribution at $21049 \mathrm{~Hz}$. 
Fig. 4.b shows the vibration nodes in the transducer, located at the red/blue borders, including the twelve nodal lines of the plate and the nodes in the brass flange and in the mechanical amplifier. As the narrow part of the mechanical amplifier is elongated $\lambda / 2$ another need appears in this narrow part as can be observed in Fig. 4.b. The increase of the length of the narrow section of the horn in half wavelength, does not affect the amplification of the displacement in the tip.

According to the simulations, the frequencies of the closest eigenmodes to the operational mode are at $19716 \mathrm{~Hz}$ and $21860 \mathrm{~Hz}$, more than $800 \mathrm{~Hz}$ separation. The mode at $19716 \mathrm{~Hz}$ (Fig. 5.a) corresponds to an extensional mode of the vibrator and a flexural mode in the plate with a combination of four and six nodal lines (4|6). In the case of the eigenmode at $21860 \mathrm{~Hz}$ (Fig. 5.b), the vibrator also exhibits an extensional mode, and the rectangular plate vibrates flexurally in a complex mode consisting of a central distorted nodal circle and nodal lines that are distorted diagonals.
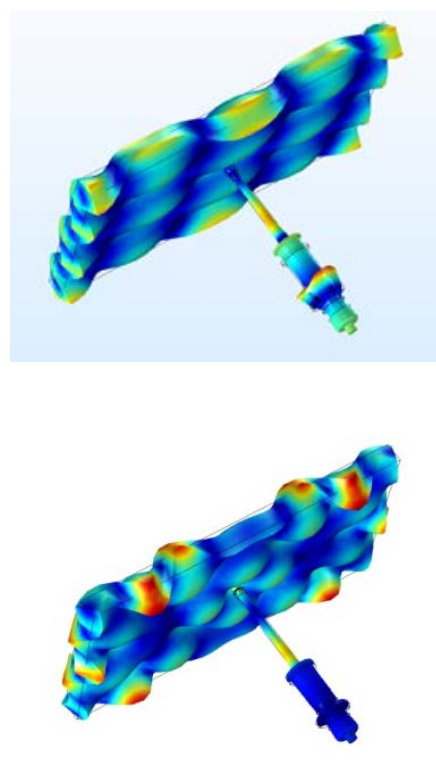

ig.5. Closest modes to the operational at $21049 \mathrm{~Hz}$ a)

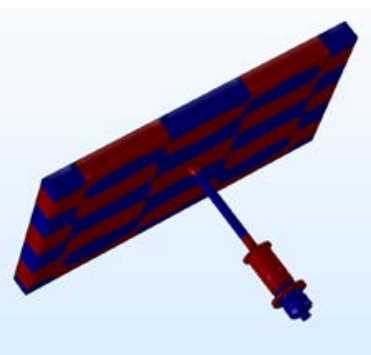

a)

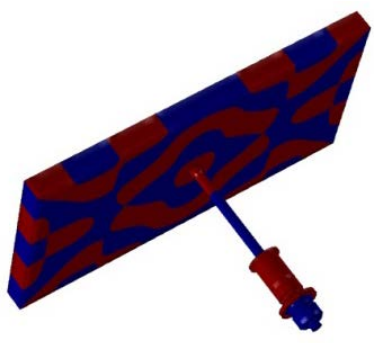

b)
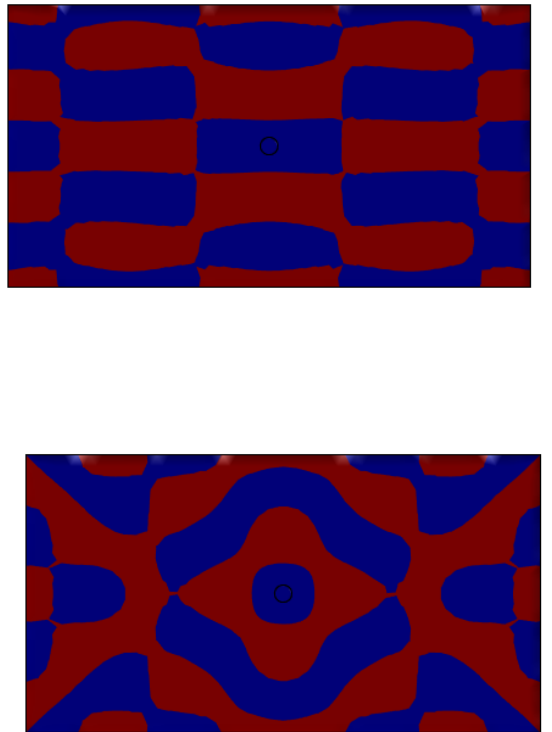

ape and node location of the mode at $19716 \mathrm{~Hz}$. b) Shape and node location of the mode at $21860 \mathrm{~Hz}$.

\section{2. - Experimental analysis - linear response regime}

An experimental modal analysis was performed to validate the numerical model. The experiments were carried out using a 3D laser vibrometer (Polytec), capable of measuring the vibration velocity in three orthogonal directions. The measurement setup is shown in Fig. 6.

The measurement process consists of defining a grid of measurement points on the surfaces of the ultrasonic vibrator and the radiating plate, sufficiently fine to identify accurately all the mode shapes in the measured frequency range. The vibration displacements in the three orthogonal axes are then measured at each grid point using the 3D laser vibrometer and these measurements are processed in ME'Scope modal analysis software in order to characterize the modes of vibration. 


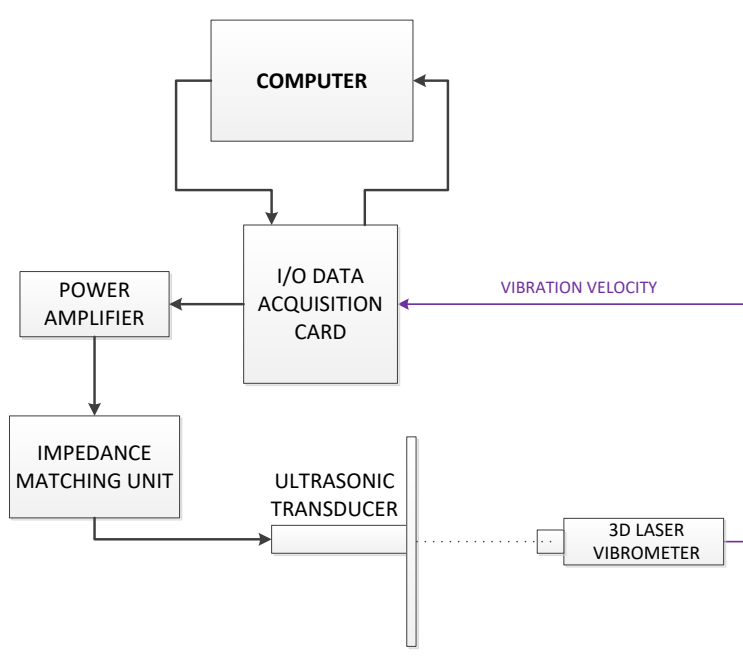

a)

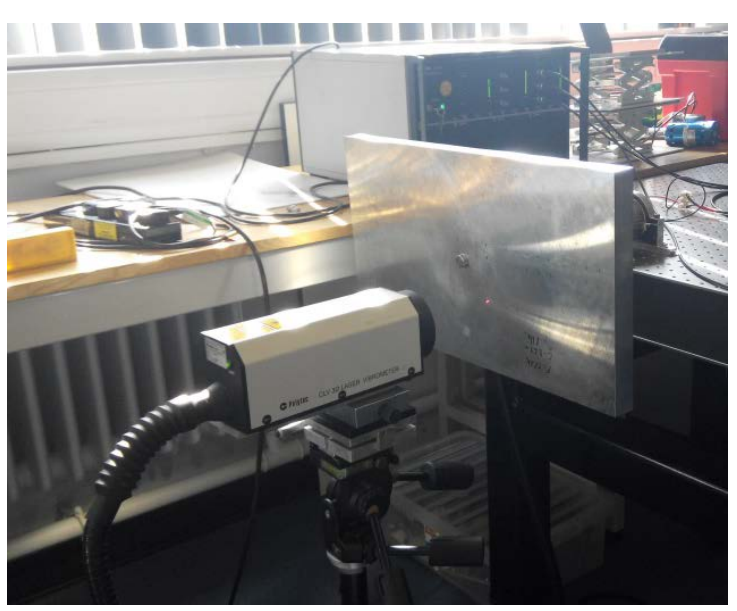

b)

Fig.6. a) Modal analysis measurement setup. b) Experimental modal analysis of the rectangular plate transducer.

The points of the grid define a wire-mesh model of the device that is used in the modal analysis software (ME'Scope) to represent the vibrational shapes at each modal frequency. In this case, the cylindrical parts of the transducer like the steel case, which protects the Langevin-type sandwich and the mechanical amplifier, would require too many measurement points (or grid points) to look like cylinders in the model. Considering that the case is not supposed to vibrate with the rest of the transducer because it is isolated from the rest of the constitutive elements, only four points in the perimeter were considered. In the case of the mechanical amplifier, also four points were considered taking into account that they are enough to identify flexural and torsional modes. For this reason, both the case and the mechanical amplifier look like square prisms instead of cylinders in the model shown in Fig. 7.

In this case, 448 grid points are defined for the rectangular plate transducer, configured as $28 \mathrm{x}$ 16 points on the plate surface. The ultrasonic vibrator has 56 grid points. The grid for the whole plate radiator transducer is shown in Fig. 7.

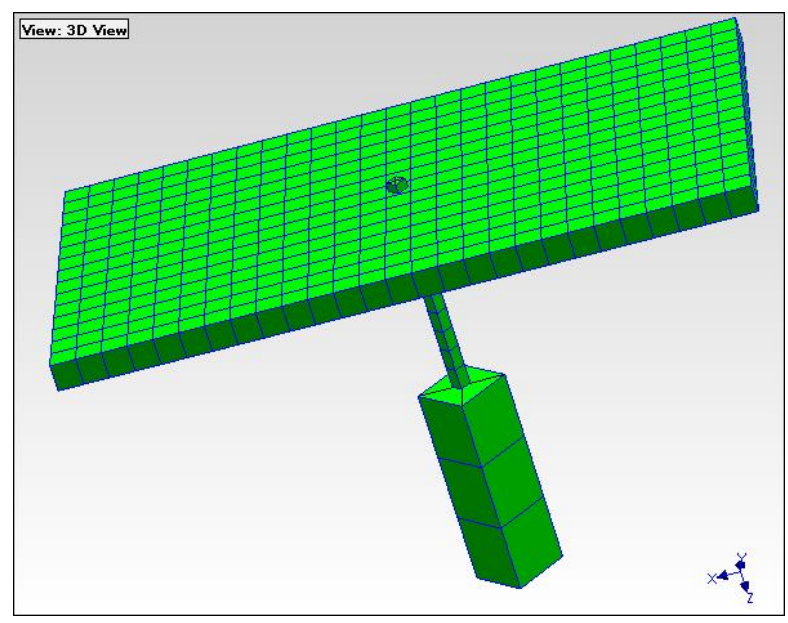

Fig.7. ME'Scope model of the rectangular plate transducer.

Fig. 8 shows the spectral distribution of the modal frequencies in the range $0-90 \mathrm{kHz}$, particularly showing the high modal density in the $15-30 \mathrm{kHz}$ range which contains the operational mode. A zoom in on a narrower range near the operational frequency (Fig. 8 b) contains the two closest modal frequencies identified in the FEM. This representation is done in terms of CMIF (Complex Mode Indicator Function), a dimensionless magnitude used to plot the eigenvalues obtained from a FRF (Frequency Response Function). 


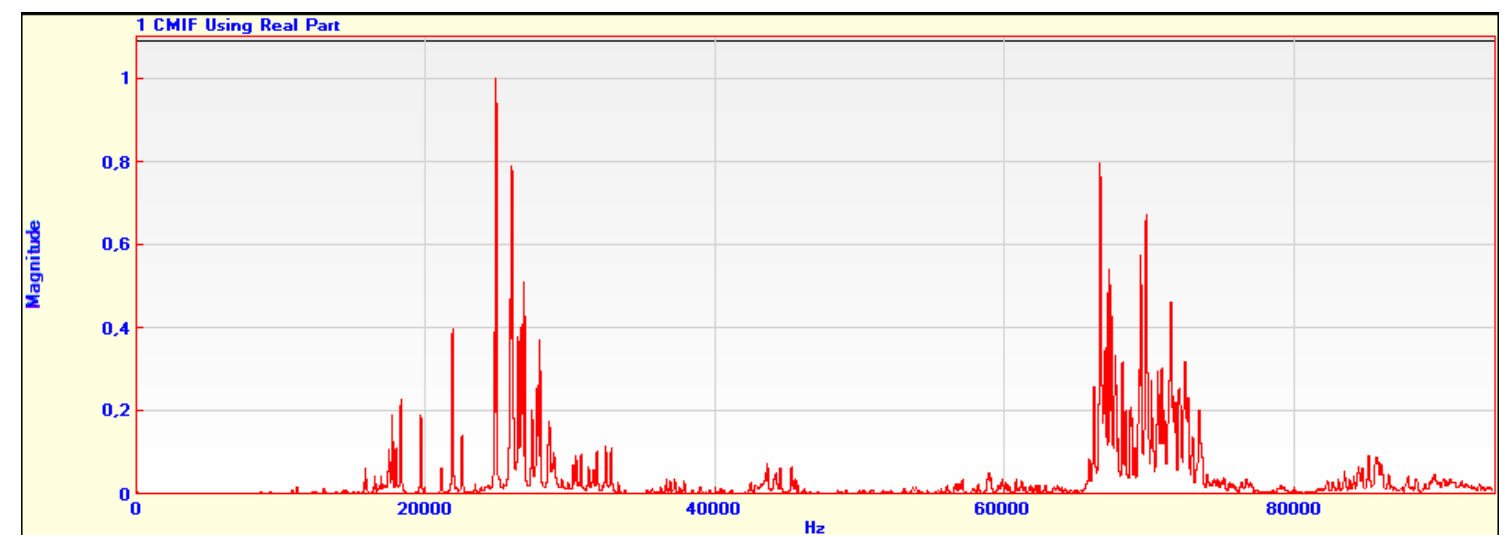

a)

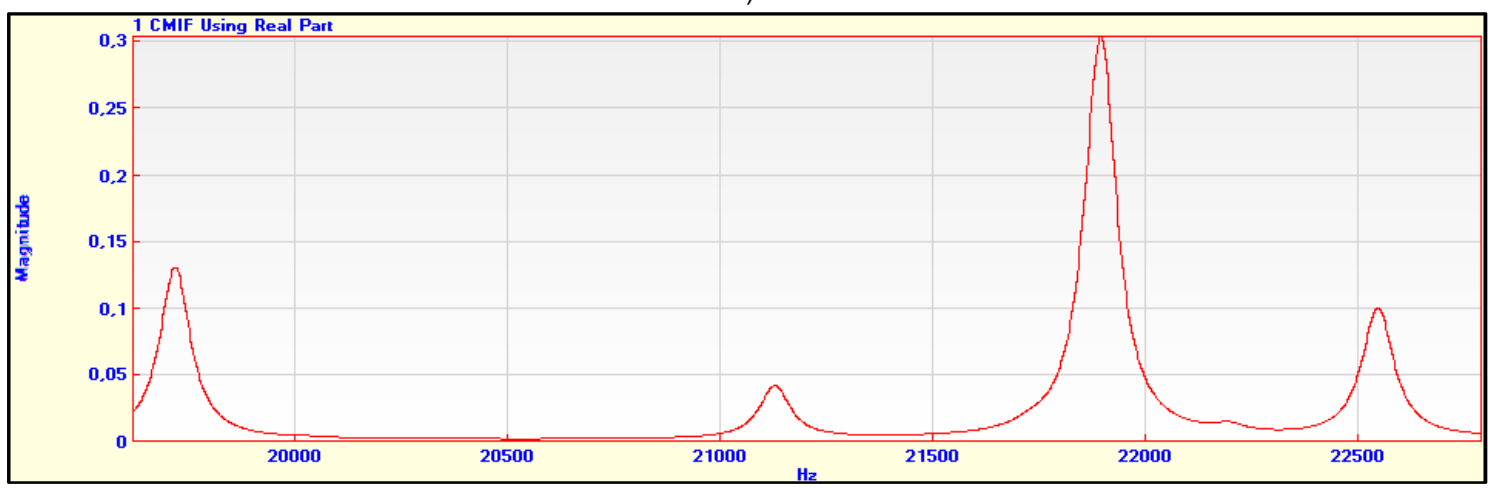

b)

Fig.8. Spectral distribution of the modal frequencies of the rectangular plate transducer. a) Spectrum between $1 \mathrm{kHz}$ to $90 \mathrm{kHz}$. b) Zoom around the operational frequency.

The frequency of the operational vibration mode is measured at $21130 \mathrm{~Hz}$, with a difference of less than $0.5 \%$ from the FEM prediction of $21049 \mathrm{~Hz}$. Although the other near modes show higher CMIF magnitude than the operational one, the required mode with 12 nodal lines appears at $21130 \mathrm{~Hz}$, as can be observed in Fig. 9.

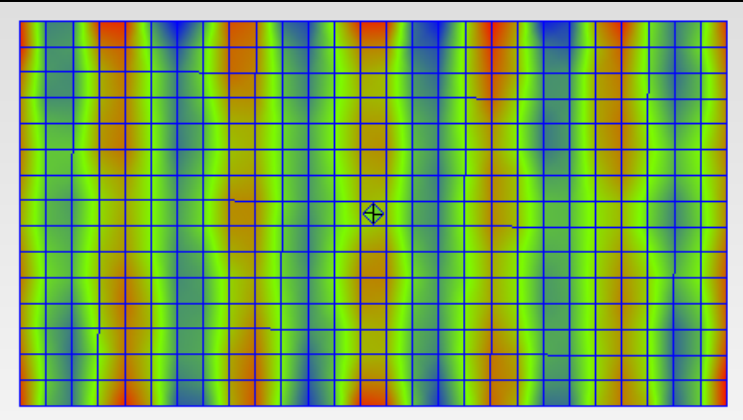

a)

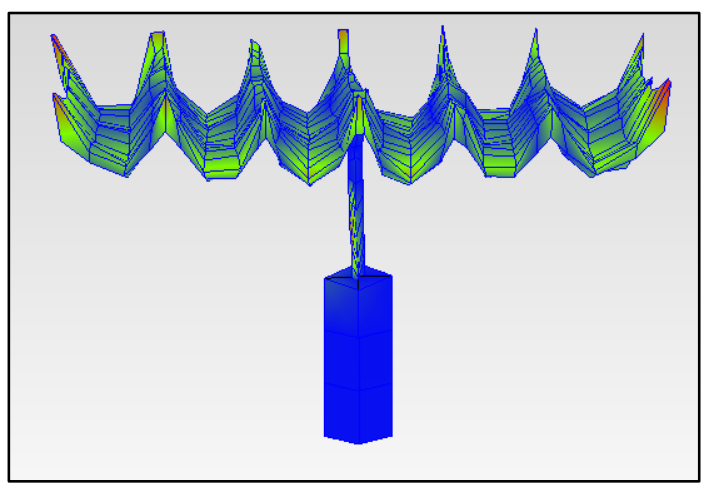

b)

Fig. 9. Twelve nodal-line operational mode for the rectangular plate transducer at $21130 \mathrm{~Hz}$. a) Node distribution. b) Mode shape.

The closest mode frequency is measured at $21898 \mathrm{~Hz}$, about $770 \mathrm{~Hz}$ from the operational mode frequency and a sufficient separation to avoid interaction between the two modes. This mode and the next closest in frequency are shown in Fig. 10. The mode at $19720 \mathrm{~Hz}$ (Fig. 10.a) consists on an extensional mode in the mechanical amplifier and a flexural mode in the rectangular plate (4|6), exactly matching the FEM predicted modal frequency and shape. The other close mode, at $21898 \mathrm{~Hz}$ is an extensional mode in the vibrator and a complex flexural mode in the rectangular plate (Fig. 10.b), again matching the FEM predictions. The modal analysis also supports the conclusion from the FEM that no modal coupling should be expected in the plate radiator 
transducer in operation since the frequency separation of the modes is sufficient to ensure no modal interaction.

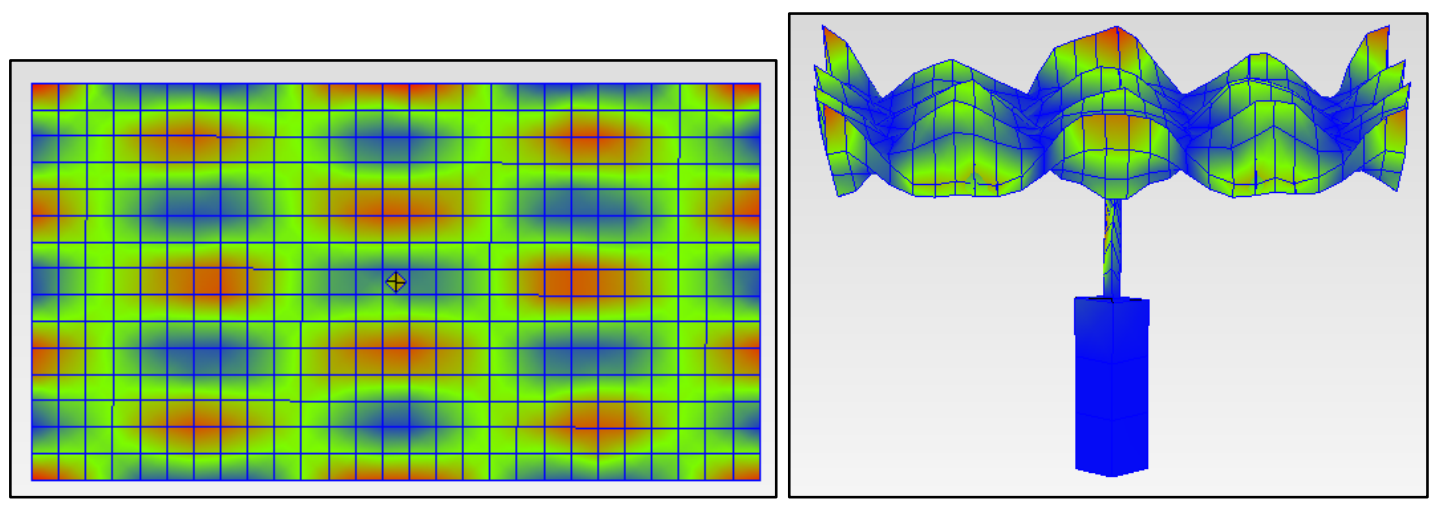

a)
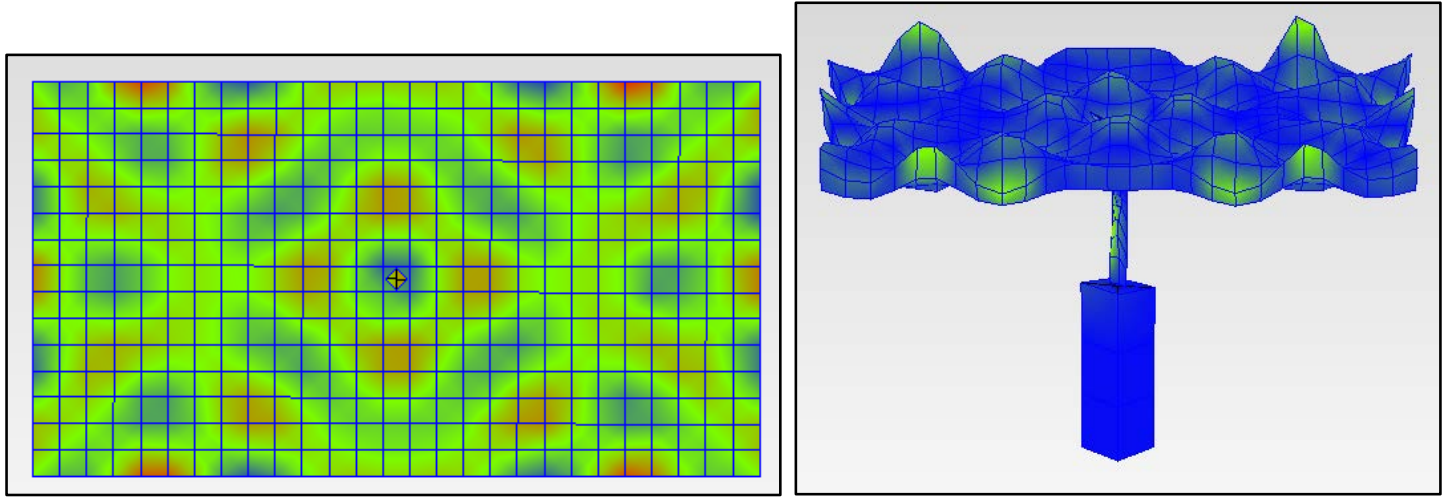

b)

Fig.10. Closest modes to the operational at 21130. a) Shape and node location of the mode at $19720 \mathrm{~Hz}$. b) Shape and node location of the mode at $21898 \mathrm{~Hz}$.

\section{4. - Dynamic characterization of the rectangular plate transducer}

Two sets of dynamic characterization experiments were carried out: stability tests and nonlinear dynamic response measurements.

\section{1. - Stability tests}

One of the main factors limiting the maximum acoustic energy that an airborne power ultrasonic transducer can transfer into a gas medium is the power capacity of the transducer and the fatigue limit below which tuned components must operate. The aim of stability tests is to determine the behavior of the ultrasonic system when operating at high power for a long duration, in terms of both fatigue and modal interactions. When an APU transducer operates continuously, eventually the constitutive parts can experience effects due to fatigue. In fact, fatigue is a term that applies to changes in properties of materials subjected to cyclic mechanical stresses or strains and can lead to component fracture and failure. Experimental tests [31] have shown that the maximum stress that can be applied without danger of ultrasonic fatigue is approximately $30 \mathrm{MPa}$ for aluminum and $200 \mathrm{MPa}$ for titanium alloy.

For repeated operation, it has been stated that, although aluminum continues degrading with use, the fatigue limit is $5 \cdot 10^{8}$ cycles [27], meaning that if no undesired behavior appears before this limit, no other effect is expected due to fatigue.

For all power ultrasonic transducers, there is a shift in the tuned resonance frequency during continuous operation due to heating of the ceramics and other components. It is then possible for 
energy to be shared with other modes if the modal density of the transducer is high. This then reduces in the efficiency of the system and increases the risk of fatigue failure.

The ultrasonic signal generation system used to carry out the stability tests consists of a dynamic resonance frequency control unit (ultrasonic controller), to give adjustable continuous power output at the resonance frequency of the transducer by keeping the voltage $(\mathrm{V})$ and current $(\mathrm{I})$ signals in phase, and tracking it when this frequency shifts during operation [32,33]. The controller operates as a finely tuned electronic signal generator that sends the excitation signal to a broadband power amplifier and then to the transducer through an impedance matching unit to allow maximum energy transfer between the electronics and the transducer.

The electrical response of the transducer can be measured at the output of impedance matching unit by sampling the voltage and current signals that are sent back to the controller. This displays information about voltage, current, power, impedance and phase. Additionally, an oscilloscope displays the frequency spectrum of this response, which can be used to check any possible modal interaction. The block diagram of the power electronic system is shown in Fig. 11.

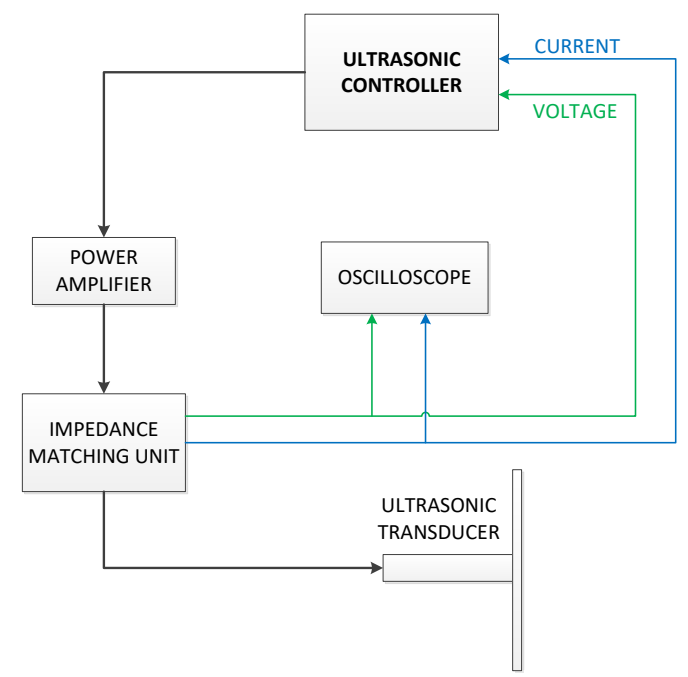

Fig.11. Block diagram of the power electronic system for stability tests.

The stability tests consist of keeping the system working for long time (up to 100 hours) at different power levels in the range $50 \mathrm{~W}-400 \mathrm{~W}$. For each test, the evolution of the resonance frequency, the power and the spectrum displayed on the oscilloscope are all monitored. A summary of the final results at the end of each stability test is presented in Table 3.

Table 3. Results after stability tests.

\begin{tabular}{|c|c|c|c|c|c|}
\hline Power (W) & Frequency (Hz) & Voltage (V) & Current (mA) & $\begin{array}{c}\text { Impedance IZI } \\
(\Omega)\end{array}$ & Time (h) \\
\hline $\mathbf{5 0}$ & 21141 & 209 & 237 & 883 & 10 \\
\hline $\mathbf{1 0 0}$ & 21140 & 293 & 343 & 847 & 25 \\
\hline $\mathbf{2 0 0}$ & 21088 & 391 & 490 & 819 & 50 \\
\hline $\mathbf{2 5 0}$ & 21080 & 452 & 566 & 778 & 50 \\
\hline $\mathbf{3 0 0}$ & 21076 & 463 & 677 & 685 & 50 \\
\hline $\mathbf{3 5 0}$ & 21071 & 492 & 727 & 697 & 100 \\
\hline 400 & 21065 & 523 & 763 & 683 & 25 \\
\hline
\end{tabular}


Table 3 shows the stable behavior of the main electrical characteristics of the transducer at the different power applied and working for 100 hours in the higher power regime (about $400 \mathrm{~W}$ ). The whole operation corresponds to $7.6 \cdot 10^{9}$ cycles without any signs of ultrasonic fatigue or saturation. This number of cycles is much higher than $5 \cdot 10^{8}$, number established as sufficient for fatigue tests for aluminum. The power capacity of the APUT can also be observed in Fig. 12, which represents the relationship between the supplied excitation voltage and the electrical power.

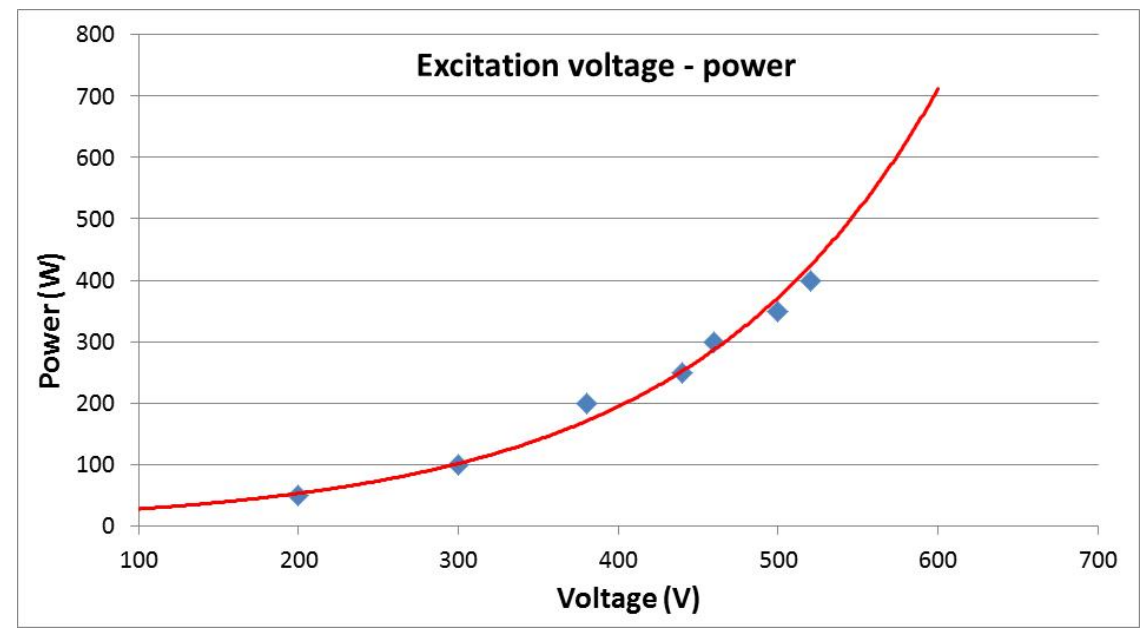

Fig.12. Relation between excitation voltage and power.

\section{2. - Nonlinear characterization}

The nonlinear dynamic characterization is be performed by carrying out a frequency sweep of the transducer amplitude around the tuned resonance frequency, repeated for different excitation levels [34, 35]. For this purpose, a power characterization system was developed [23], capable of measuring the nonlinear dynamics of APU transducers, taking into account electrical, acoustical, thermal and vibrational aspects simultaneously. The experiments were carried out in this study using this nonlinear characterization system, whose block diagram is shown in Fig. 13.

The main goal of this characterization system is to perform multiple synchronized analysis tasks, including signal acquisition, signal analysis, characterization, control, monitoring and data logging. This system provides some additional capabilities such as selection of maximum allowed values of current, voltage or temperature; resonance tracking software applying conventional algorithms (maximum power and minimum phase). A further capability of this system is the virtual compensation of the parallel capacitance $\left(C_{p}\right)$ when tracking resonance. This means that it is not necessary to connect the required inductance $(\mathrm{L})$, reducing the distortion in the impedance matching unit. All these software solutions provide flexibility and fast reprogramming when modifying any parameter (e.g. excitation voltage, tolerances, $C_{p}$ compensation). 


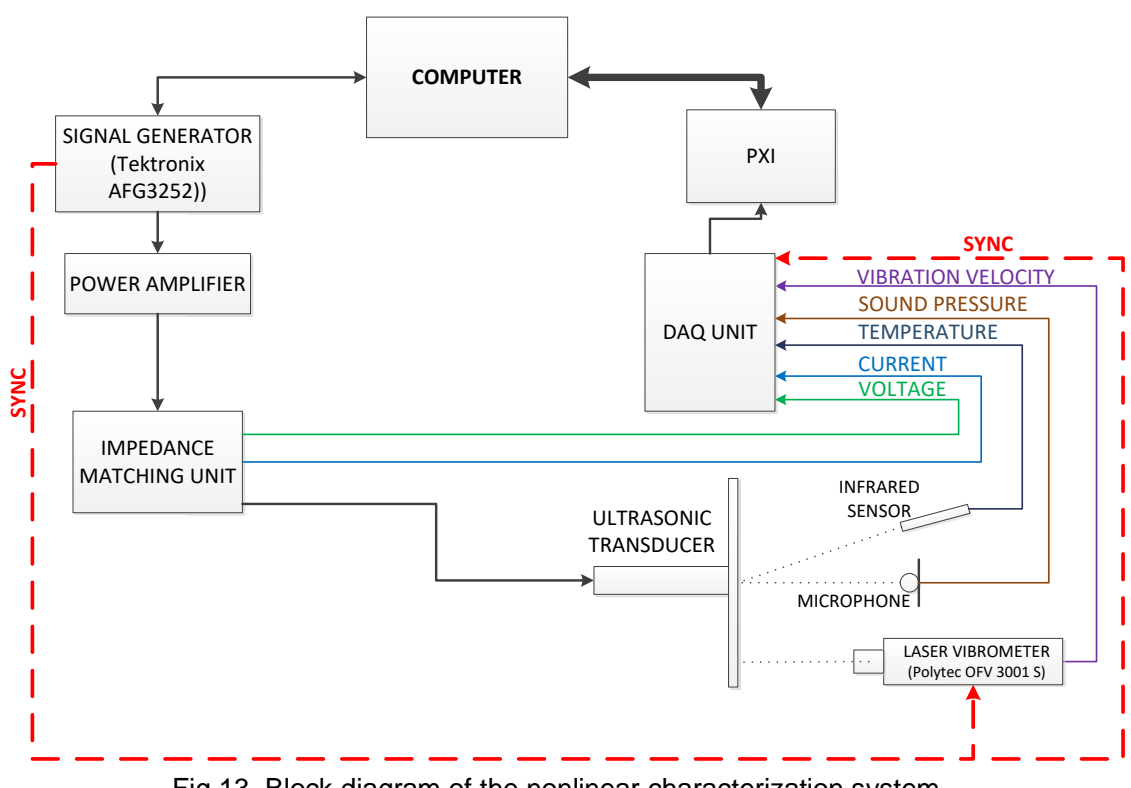

Fig.13. Block diagram of the nonlinear characterization system

The response of the transducer was measured electrically, by sampling voltage and current at the output of the impedance matching unit. The mechanical response was measured with a 1D laser vibrometer (Polytec OFV 3001S) that measures the out-of-plane component of the vibration velocity of the plate surface at an antinode of the plate operating mode. An infrared sensor was also used in the experiment to monitor the temperature of the piezo-ceramic stack.

The nonlinear characterization of the plate transducer includes a broadband sweep response, forward and backward sweep response measurements in a narrow band around the resonance frequency, FFT analysis and temperature monitoring.

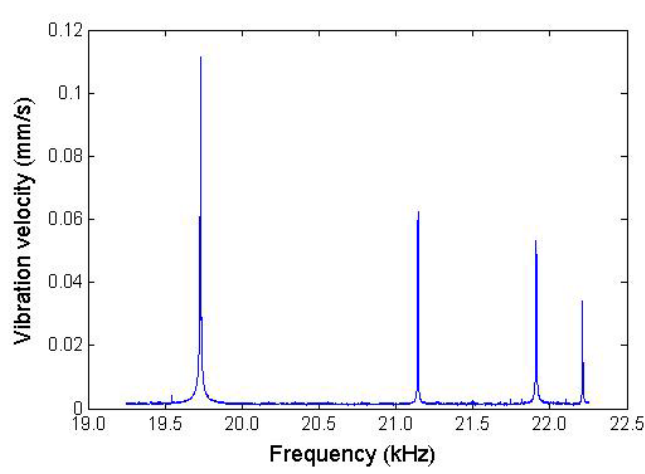

a)

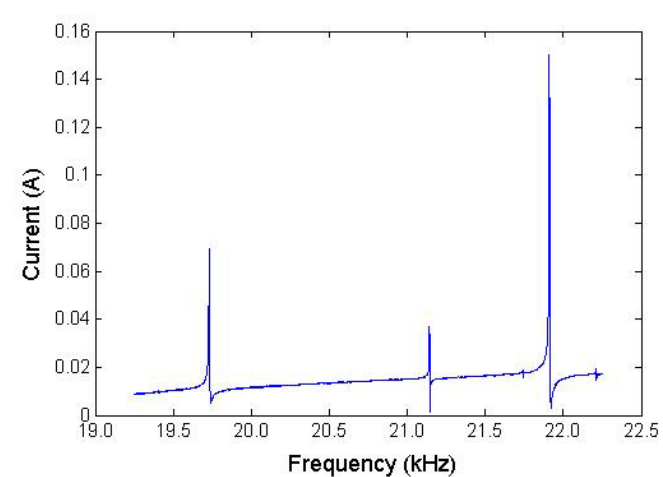

b)

Fig.14. a) Vibration velocity in the rectangular plate. b) Electric response of the transducer (current).

Fig. 14.a shows a low power ( $5 \mathrm{~W}$ ) sweep in a $19-22.5 \mathrm{kHz}$ range, for vibration velocity and electric current, identifying the operational mode frequency and other close modal frequencies, as found in the modal analysis. In Fig. 14.b, it is also seen the current peaks at the modal frequencies.

To fully characterize the dynamic behavior of the system, it is necessary to measure frequency drift due to the temperature increasing in the ceramics, as well as investigating the appearance of hysteretic effects and softening or hardening nonlinear responses at increasing excitation levels.

The procedure of the frequency sweeps consists of incrementing the excitation frequency upwards and downwards in a narrow frequency band through resonance. The sweeps were repeated for increasing excitation voltage in increments up to $225 \mathrm{~V}$. The experiments were 
carried out using a continuous wave excitation and a burst excitation signal. This allowed for distinguishing between responses showing the frequency drift associated with heating in the piezoceramics (continuous wave excitation) and those associated with nonlinear dynamics of the transducer (where heating effects are eliminated by adopting burst excitation). Fig. 15 shows the results presenting the measured vibration velocity using the $1 \mathrm{D}$ laser vibrometer, pointing at a maximum displacement area of the plate.

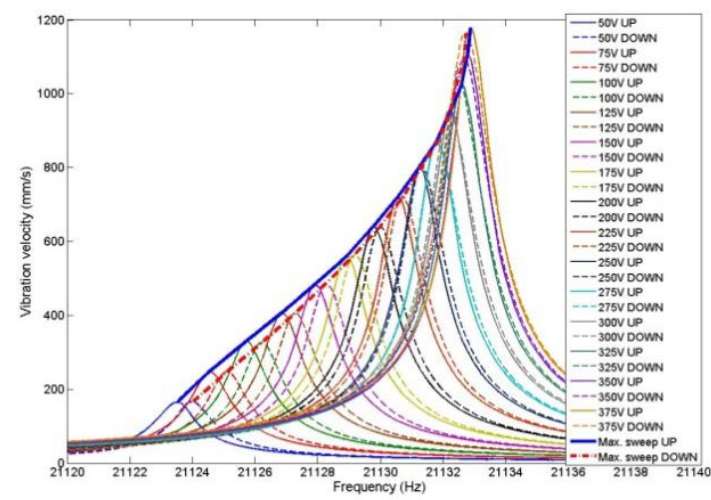

a)

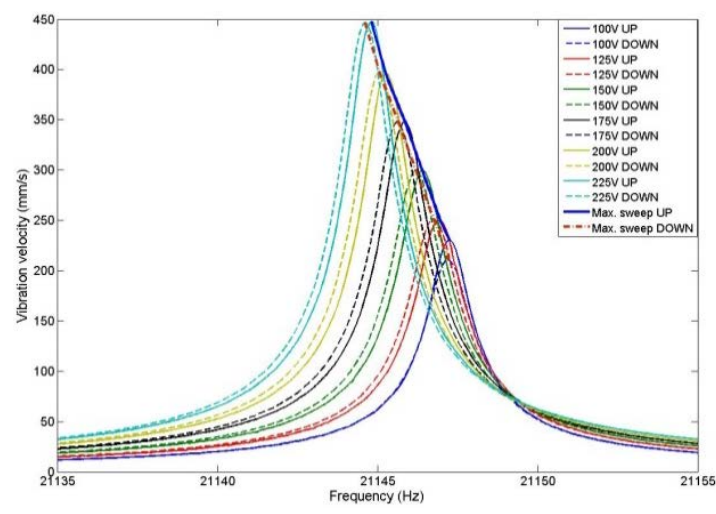

b)

Fig.15. 2D sweeps. a) Continuous signal excitation. b) Burst excitation

The measured frequency shift between $100 \mathrm{~V}$ and $225 \mathrm{~V}$ excitation level is only $5 \mathrm{~Hz}$, which is negligible. The measured responses are highly linear, exhibiting symmetry near overlap in the forward and backward sweep responses and no evidence of a bending backbone curve that would signify a softening or hardening nonlinearity Moreover, no hysteretic responses have been found, meaning that this is a very stable system.

During the frequency sweep measurements, the temperature was measured at the union between the flat rectangular radiator plate and the mechanical amplifier, and this remained stable at $22{ }^{\circ} \mathrm{C}$ through all the experiments. To further evaluate the temperature increase in the transducer, a resonance tracking experiment was undertaken, with the transducer operating for two hours at a constant excitation of $200 \mathrm{~W}$. Fig. 16 shows that the temperature was increased by less than 4 ${ }^{\circ} \mathrm{C}$ and was stabilizing after two hours.

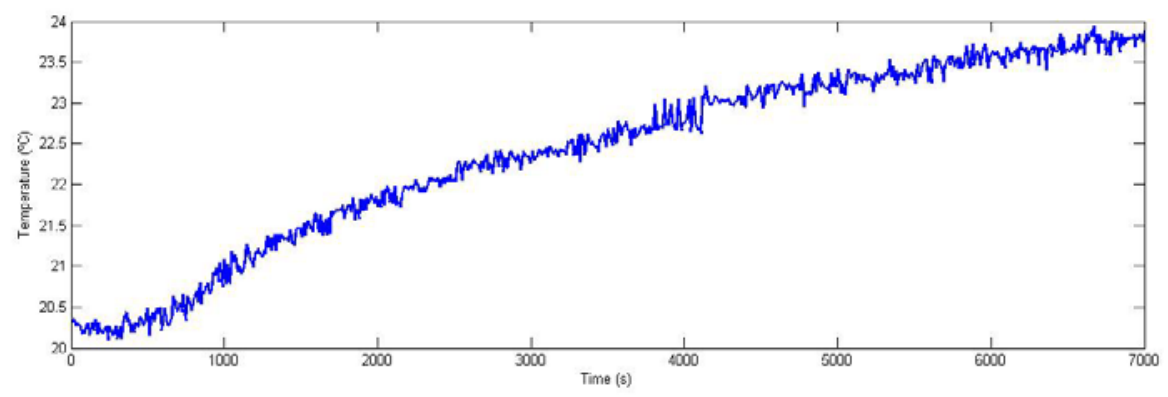

Fig.16. Evolution of temperature in a high power regime operation.

Overall, the nonlinear characterization has shown a very stable transducer exhibiting no adverse influences of temperature, modal interactions or nonlinear responses.

\section{5. - Design of the reflector system}

As determined, the flat plate radiator transducer exhibits highly stable and linear behavior up to the operating power levels. However, using a flat plate means that, unlike conventional stepped plate radiators, the reflectors must be accommodated in a separate system. To provide comparable performance, the reflectors must enable coherent radiation patterns with high sound 
pressure levels (more than $150 \mathrm{~dB}$ in the near field) and an electroacoustic efficiency around $70 \%$.

Conventionally, in order to generate a coherent ultrasonic field from a plate radiator transducer, stepped geometries are incorporated at the nodal lines of the plate. The height of each step must be a half-wavelength in the propagating medium. The stepped plate transducer with rectangular radiator has shown modal interaction problems at high power operation [14]. These are probably due to the higher density mode of the stepped rectangular plates. Therefore, the design based on a flat plate radiator with reflectors represents an alternative for power operation with rectangular plates.

To solve this, a system, that is composed of a flat rectangular plate transducer and a stepped reflector structure, is presented [26]. The reflectors form a 45 degrees angle with the two sides of the rectangular plate in order to double the radiating surface of the transducer. In this way, the acoustic energy from both sides of the radiator can be used to obtain the desired effects if a directional or focused acoustic field is reached in the medium, increasing also the electroacoustic efficiency of the system.

The steps are located in the reflector structure instead of in the plate, and the height of the steps depends on the wavelength of the sound in the medium. As shown in Fig. 17.a, the ultrasonic field generated by both faces of the plate radiator propagates in anti-phase to the stepped reflectors. The alternative addition of steps implies that the waves in phase have to travel half wavelength more than the waves in anti-phase. This fact allows an in-phased ultrasonic field after the reflector system.

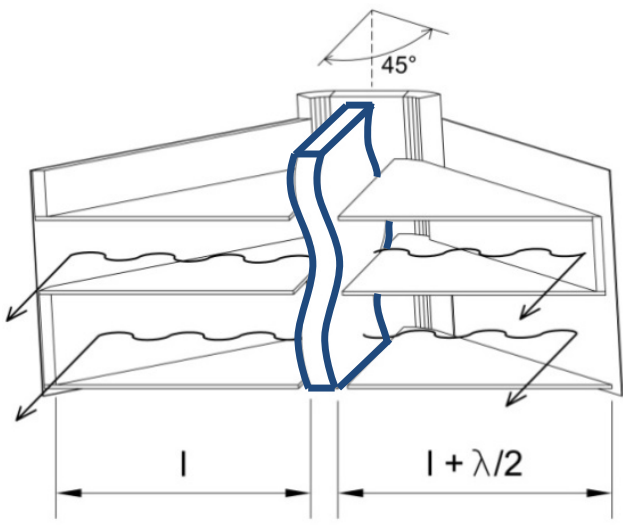

a)

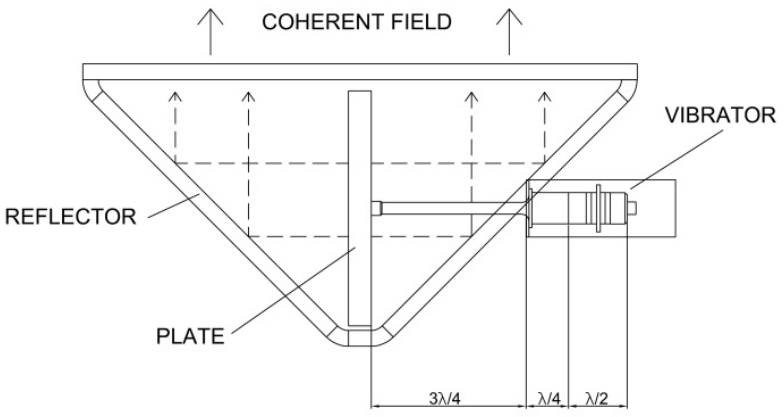

b)

Fig.17. Food dehydration system with an airborne power ultrasonic transducer and a reflector system; a) Description of the generation of a coherent field after reflections in the stepped reflectors. b) Plant view of the airborne power ultrasonic transducer and the reflector system.

The advantage of lengthening the mechanical amplifier in the plate transducer by a halfwavelength, $\lambda / 2$, in the narrow section is now seen in Fig. 17.b. With this extension, the case remains outside the reflectors system and the interferences are therefore minimized.

The rear face, as well as the front face, of the plate is radiating ultrasonic waves to the reflectors. Therefore it is possible for the vibrator to interfere with the field between both elements. The operational frequency of the ultrasonic transducer is $21 \mathrm{kHz}$ in air, with a wavelength of $16 \mathrm{~mm}$. The narrow section of the mechanical amplifier has a diameter of $10 \mathrm{~mm}$, which is about a halfwavelength of the ultrasonic field in air. This relationship between the wavelength and diameter of the narrow section of the horn minimizes the influence of this element in the ultrasonic field. The wider section of the mechanical amplifier has a diameter of $20 \mathrm{~mm}$, and could therefore produce strong interference in the ultrasonic field generated by the plate. The reason why the 
narrow section has been lengthened is to keep this wider section outside the transducer-reflector system.

Once the global behavior of the active part of the system has been proved to be successful, future experimental acoustic analysis of the whole system with reflectors will be taken in order to prove that it is capable of generate a coherent field with high sound pressure levels.

\section{6. - Conclusions}

A new airborne power ultrasonic rectangular plate transducer for airborne applications has been designed, built and characterized to determine its dynamic behavior. The global behavior of the plate transducer has been analyzed by numerical and experimental modal analysis at low power and by experimental characterization of stability and nonlinear dynamics at high power operating conditions. The designed APU transducer has a very high quality factor $(Q \approx 13000)$, a narrow bandwidth $(1.59 \mathrm{~Hz})$, an electro-acoustical efficiency of about $80 \%$ and a resonance frequency around $21100 \mathrm{~Hz}$.

According to the results obtained with both methods (numerical and experimental), the transducer has high modal density around the tuned resonance frequency but does not exhibit any modal interactions at low power.

The stability tests showed that the transducer is a highly resonant system, with a high power capacity and negligible temperature increases when operating at high power for long durations.

Finally, the frequency sweep results showed a negligible frequency shift for both continuous and burst signal excitation and no other nonlinear responses were detected up to an excitation level of $375 \mathrm{~V}$.

The plate transducer is intended to be the active part of an innovative system where the passive element will be a separate stepped reflector system that enables the generation of a coherent ultrasonic field in free field conditions. Future research will seek to characterize the acoustic field generated by the ultrasonic system with reflectors in a low density gas medium.

\section{Acknowledgments}

This work has been supported by the project DPI2012-37466-C03-01 funded by the Spanish Ministry of Economy and Competitiveness.

The experimental study has been carried out in the facilities of the School of Engineering of the University of Glasgow. The stay in Glasgow has been supported by the Spanish Ministry of Economy and Competitiveness, with the grant EEBB-I-16-11728. We would like to thank Dr. Andrew Mathieson and Dr. Andrew Feneey for their kind welcome at the School of Engineering of Glasgow University and for their assistance during the experimental campaign. We would also like to show our gratitude to Mr. Alberto Pinto for his dedication in the mechanical laboratory achieving maximum quality transducer assemblies, as important as the design for the good performance of the ultrasonic system. Finally, thanks to Mr. Alfonso Blanco for devising and implementing the nonlinear characterization system, which allows faster and more accurate experimental tasks.

\section{References}

[1] G. Rodríguez, E. Riera, J.A. Gallego-Juárez, V.M. Acosta, A. Pinto, I. Martínez, A. Blanco, Experimental study of defoaming by air-borne power ultrasonic technology, Physics Procedia, 3 (2010) 135-139. 
[2] E. Riera, I. González-Gomez, G. Rodríguez, J.A. Gallego-Juárez, Ultrasonic agglomeration and preconditioning of aerosol particles for environmental and other applications, in: J.A. GallegoJuárez, K.F. Graff (Eds.) Power Ultrasonics, Woodhead Publishing, Oxford, 2015, pp. 1023-1058. [3] S. de la Fuente-Blanco, E. Riera-Franco de Sarabia, V.M. Acosta-Aparicio, A. Blanco-Blanco, J.A. Gallego-Juárez, Food drying process by power ultrasound, Ultrasonics, 44 (2006) 523-527. [4] M. Villamiel, J. Gamboa, A.C. Soria, E. Riera, J.V. García-Pérez, A. Montilla, Impact of power ultrasound on the quality of fruits and vegetables during dehydration, Physics Procedia, 70 (2015) 828-832.

[5] J.V. García-Pérez, J.A. Carcel, A. Mulet, E. Riera, J.A. Gallego-Juarez, 29 - Ultrasonic drying for food preservation, in: J.A. Gallego-Juárez, K.F. Graff (Eds.) Power Ultrasonics, Woodhead Publishing, Oxford, 2015, pp. 875-910.

[6] J.A. Gallego-Juarez, G. Rodriguez, V.M. Acosta-Aparicio, E. Riera, Power ultrasonic transducers with extensive radiators for industrial processing, Ultrasonics sonochemistry, 17 (2010) 953-964.

[7] J.A. Gallego-Juárez, G. Rodríguez, V.M. Acosta-Aparicio, E. Riera, A. Cardoni, 7 - Power ultrasonic transducers with vibrating plate radiators, in: J.A. Gallego-Juárez, K.F. Graff (Eds.) Power Ultrasonics, Woodhead Publishing, Oxford, 2015, pp. 159-193.

[8] A. Mathieson, A. Cardoni, N. Cerisola, M. Lucas, Understanding nonlinear vibration behaviours in high-power ultrasonic surgical devices, Proceedings of the Royal Society A: Mathematical, Physical and Engineering Science, 471 (2015).

[9] M. Umeda, K. Nakamura, S. Ueha, Effects of vibration stress and temperature on the characteristics of piezoelectric ceramics under high vibration amplitude levels measured by electrical transient responses, Japanese Journal of Applied Physics, 38 (1999) 5581.

[10] A. Cardoni, E. Riera, J.A. Gallego-Juárez, Nonlinear response in airborne piezoelectric transducer for power ultrasonics, in: Proceedings of the 2013 International Congress on Ultrasonics (ICU 2013), Singapore, 2013, pp. 173-178.

[11] N. Aurelle, D. Guyomar, C. Richard, P. Gonnard, L. Eyraud, Nonlinear behavior of an ultrasonic transducer, Ultrasonics, 34 (1996) 187-191.

[12] K. Uchino, J. Zheng, A. Joshi, Y.-H. Chen, S. Yoshikawa, S. Hirose, S. Takahashi, J.W.C. de Vries, High Power Characterization of Piezoelectric Materials, Journal of Electroceramics, 2 (1998) 33-40.

[13] A. Cardoni, M. Lucas, M.P. Cartmell, F.C. Lim, Nonlinear and parametric vibrations in ultrasonic cutting systems, in: Materials science forum, 2003, pp. 397-406.

[14] J.A. Gallego-Juárez, E. Riera, V.M. Acosta-Aparicio, Modal interactions in high-power ultrasonic processing transducers, AIP Conf. Proc., 1022 (2008) 595-604.

[15] A. Mulet, J.A. Cárcel, N. Sanjuán, J. Bon, New food drying technologies - use of ultrasound, Food Science and Technology International, 9 (2003) 215-221.

[16] J.V. Garcia-Perez, J.A. Carcel, E. Riera, C. Rosselló, A. Mulet, Intensification of lowtemperature drying by using ultrasound, Drying Technology, 30 (2012) 1199-1208.

[17] E. Neppiras, The pre-stressed piezoelectric sandwich transducer, Ultrasonics international 1973, (1973) 295-302.

[18] C. Ranz-Guerra, R. Dominguez Ruiz-Aguirre, Composite sandwich transducers with quarter-wavelength radiating layers, The Journal of the Acoustical Society of America, 58 (1975) 494-498.

[19] A. Abdullah, M. Shahini, A. Pak, An approach to design a high power piezoelectric ultrasonic transducer, Journal of Electroceramics, 22 (2009) 369-382.

[20] E. Riera, J.V. García-Pérez, J.A. Cárcel, V.M. Acosta-Aparicio, J.A. Gallego-Juárez, Computational study of ultrasound-assisted drying of food materials, in: Innovative Food Processing Technologies: Advances in Multiphysics Simulation, Blackwell Publishing Ltd., 2011, pp. 265-301.

[21] A. Abdullah, A. Pak, Correct prediction of the vibration behavior of a high power ultrasonic transducer by FEM simulation, Int J Adv Manuf Technol, 39 (2008) 21-28. 
[22] A. Iula, F. Vazquez, M. Pappalardo, J.A. Gallego, Finite element three-dimensional analysis of the vibrational behaviour of the Langevin-type transducer, Ultrasonics, 40 (2002) 513-517.

[23] R.R. Andrés, A. Blanco, V.M. Acosta-Aparicio, E. Riera, I. Martínez, A. Pinto, New ultrasonic controller and characterization system for low temperature drying process intensification, Physics Procedia, 70 (2015) 833-836.

[24] A. Cardoni, E. Riera, A. Blanco, V. Acosta, J.A. Gallego-Juárez, Modal interactions in ultrasonic plate transducers for industrial applications, Proceedings of the Institution of Mechanical Engineers, Part C: Journal of Mechanical Engineering Science, 226 (2012) 20442052.

[25] F. Bejarano, A. Feeney, M. Lucas, Vibration characterisation of cymbal transducers for power ultrasonic applications, Journal of Physics: Conference Series, 382 (2012) 012063.

[26] J.A. Gallego-Juárez, G. Rodríguez-Corral, E. Riera-Franco de Sarabia, F. Vázquez-Martínez,

C. Campos-Pozuelo, V.M. Acosta-Aparicio, Recent developments in vibrating-plate macrosonic transducers, Ultrasonics, 40 (2002) 889-893.

[27] F. Beer, E.R. Jr. Johnston, J. DeWolf, D. Mazurek, Mechanics of Materials, McGraw-Hill Education, 2011.

[28] G.B. Warburton, The Vibration of Rectangular Plates, Proceedings of the Institution of Mechanical Engineers, 168 (1954) 371-384.

[29] A.W. Leissa, Vibration of plates, in, OHIO STATE UNIV COLUMBUS, 1969.

[30] G.W. Caldersmith, Vibrations of Orthotropic Rectangular Plates, Acta Acustica united with Acustica, 56 (1984) 144-152.

[31] C. Campos-Pozuelo, J.A. Gallego-Juárez, Limiting strain of metals subjected to highintensity ultrasound, Acta Acustica united with Acustica, 82 (1996) 823-828.

[32] A. Ramos-Fernandez, J.A. Gallego-Juarez, F. Montoya-Vitini, Automatic system for dynamic control of resonance in high power and high Q ultrasonic transducers, Ultrasonics, 23 (1985) 151-156.

[33] Y. Kuang, Y. Jin, S. Cochran, Z. Huang, Resonance tracking and vibration stablilization for high power ultrasonic transducers, Ultrasonics, 54 (2014) 187-194.

[34] E. Riera, A. Cardoni, V.M. Acosta-Aparicio, J.A. Gallego-Juárez, B.B. Linde, J. Paczkowski, N. Ponikwicki, Nonlinear behaviour of power ultrasonic transducers for food processing, in: AIP Conf. Proc., 2012, pp. 345.

[35] E. Riera, A. Cardoni, A. Blanco, V.M. Acosta-Aparicio, J.A. Gallego-Juárez, Characterising the nonlinear dynamics of power ultrasonic systems, in: Proceedings 39th International Congress on Noise Control Engineering, Inter noise, Lisbon, 2010. 